\title{
Fabrication of Hollow Maxillary Complete Denture Using Silicon Putty and Double Flask Technique
}

\author{
Mohit Bhatnagar ${ }^{1}$, Gagan Khanna², Pramiti Saxena ${ }^{3}$, Arti Singh ${ }^{4}$, Veenuka Sharma ${ }^{5}$ \\ 1, 2,4,5 Department of Prosthodontics, Crown and Bridge, Divya Jyoti College of Dental Sciences \\ and Research, Modinagar, Uttar Pradesh, India. ${ }^{3}$ Department of Prosthodontics, ITS Dental \\ College, Hospital and Research Centre, Greater Noida, Uttar Pradesh, India.
}

\section{INTRODUCTION}

This article describes the fabrication of a hollow maxillary complete denture using silicone putty and a double flask technique. A successful complete denture directly depends on retention, stability and support accompanied by good aesthetics. However, in case of atrophic edentulous ridges the challenge lies in the fact that there is a significantly lesser denture bearing area that can lead to a number of problems. The most important of these is an increased interarch space resulting from excessive resorption of upper or lower arch. This can lead to a resulting heavy complete denture that further deteriorates the bone condition due to excessive and continuous load, accelerating bone resorption. Moreover, there is no effective support to deal with any kind of harmful lateral forces and this can lead to dislodgment of the denture.

The operator's efficiency lies in applying the basic principles of fabricating a denture and innovating various steps to counter critical situations. Various methods such as asbestos, silicone putty, modelling clay, thermocol, salt, wax have been used for creating a hollow space inside the denture. Out of the various methods that have been used for fabrication of a hollow space inside a complete denture, silicone putty method is one of the more convenient and accurate method for achieving the objective, thereby rendering a lighter prosthesis.

A hollow maxillary denture is highly advantageous as it reduces the weight of acrylic resin, thereby preserving the residual alveolar ridge. It also helps in stability of the denture by indirectly reducing the lateral forces.

\section{PRESENTATION OF CASE}

A 54-year-old male patient reported to the Department of Prosthodontics, Crown and Bridge, with a chief complaint of having no teeth in upper and lower jaws since 4 years. On taking further history, it was found that the teeth were lost due to periodontal reasons.

After the diagnostic mounting, it was observed that there was an excessive inter ridge distance and an increased height of the maxillary rim that could have resulted in a heavy maxillary denture. Hence, a hollow maxillary complete denture was planned to tackle the problem.

In case of excessive residual ridge resorption, various other modifications such as the use of neutrocentric or lingualized occlusion, and modified impression techniques can also be used in combination.
Corresponding Author: Dr. Mohit Bhatnagar, H 37, Sector 22, Noida, Gautam Buddha Nagar, Uttar Pradesh - 201301, India.

E-mail: drmohitbhatnagar93@gmail.com

DOI: $10.14260 / \mathrm{jemds} / 2021 / 602$

How to Cite This Article:

Bhatnagar M, Khanna G, Saxena P, et al. Fabrication of hollow maxillary complete denture using silicon putty and double flask technique. J Evolution Med Dent Sci 2021;10(34):2948-2950, $10.14260 / \mathrm{jemds} / 2021 / 602$

Submission 31-12-2020,

Peer Review 19-06-2021,

Acceptance 26-06-2021,

Published 23-08-2021.

Copyright (C) 2021 Mohit Bhatnagar et al. This is an open access article distributed under Creative Commons Attribution License [Attribution 4.0 International (CC BY 4.0)] 


\section{DISCUSSION OF MANAGEMENT}

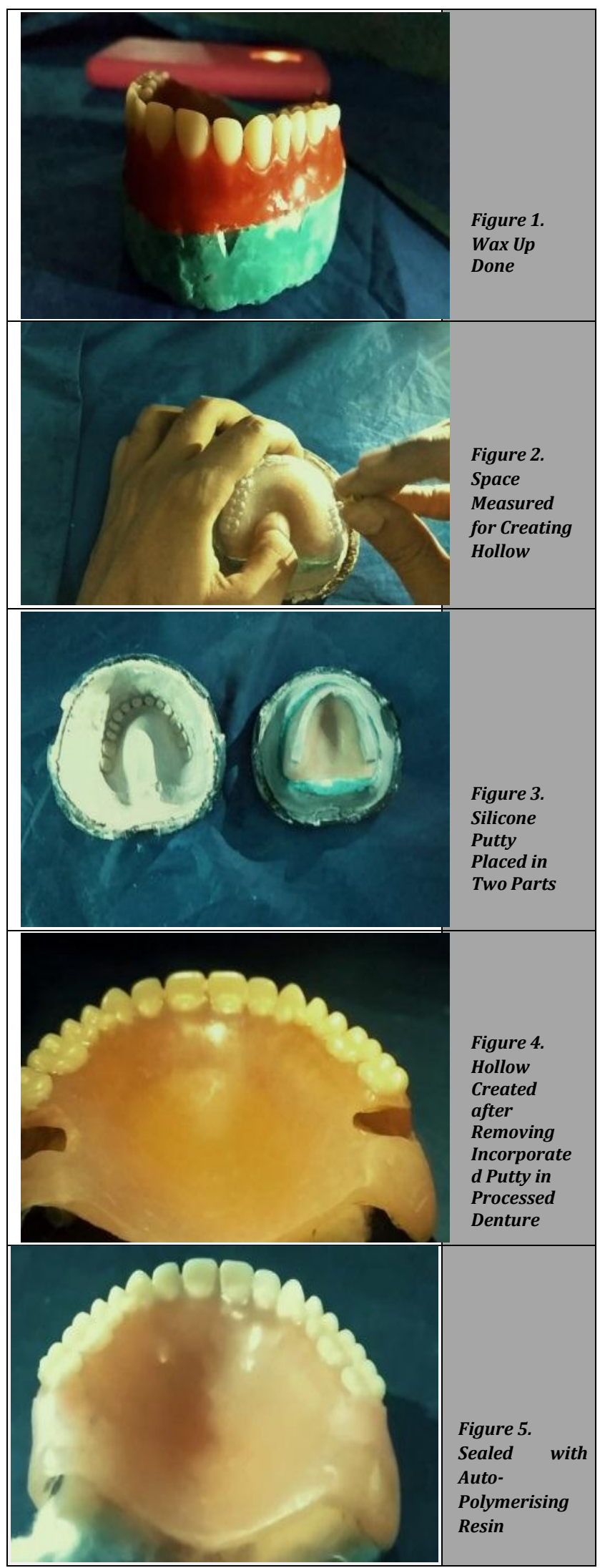

1. All the steps of fabricating a regular denture to the trial denture stage were followed and wax up was completed. (Figure 1)

2. The land area of the cast was indexed so that the following thermoplastic template could fit properly.
3. The trial denture was duplicated with irreversible hydrocolloid (Tropicalgin; Zhermack) and the impression was poured in dental stone (Kalabhai Karson Pvt Ltd).

4. A clear template of the stone cast was made using a 1.0 mm thermoplastic sheet (Leone S.p.a).

5. Regular processing steps were carried till the dewaxing stage.

6. The master cast was hence in the drag; a second flask was used to invest the two layers of baseplate wax (Prevest Denpro) which were adapted on this definitive cast followed by another dewaxing. This was done to have the heat processed baseplate portion of the denture. The cope and second drag were used to process heat - polymerized acrylic resin (Dental Products of India Ltd.).

7. Deflasking was done, and the processed baseplate was not separated until the thermoplastic template was seated on the master cast. The indices in the land area served as guides for reference. Now the tentative space between the matrix and the baseplate was measured with an endodontic file (Dentsply Maillefer) with a rubber stop. (Figure 2)

8. Condensation Sillicone based putty (Zetaplus, Zhermack) was mixed and adapted to the heat cured baseplate according to the thickness calculated earlier. Two $\mathrm{mm}$ of space was given over the artificial teeth to prevent chances of fracture. The putty matrix was fixed in two parts to the acrylic resin using cyanoacrylate adhesive (Loctite 495, Henkel adhesives technologies, India). (Figure 3)

9. This was followed by processing of the denture using the original cope. Deflasking and finishing of the denture was done.

10. The denture base distal to the most posterior teeth was drilled on both sides to make openings for retrieval of the putty matrix. The silicone putty was removed by scraping with a sharp instrument preferably an orthodontic wire. (Figure 4)

11. Putty was removed and 2 covers were fabricated using autopolymerizing resin (Dental Products of India Ltd.)

12. The cavity was cleaned and disinfected (Cidex OPA, Advanced Sterilization Products, Johnson \& Johnson Medical, Skipton, UK). This was followed by sealing of the holes using autopolymerizing resin (Dental Products of India Ltd.) (Figure 5).

13. The denture was immersed in water to check for the successful sealing.

\section{DISCUSSION}

A successful complete denture directly depends on retention, stability and support accompanied by good aesthetics. However, in case of atrophic edentulous ridges the challenge lies in the fact that there is a significantly lesser denture bearing area that can lead to a number of problems. The most important of these is an increased interarch space resulting in a heavy complete denture that further deteriorates the bone condition due to excessive and continuous load, accelerating bone resorption. Various methods such as asbestos, silicone 
putty, modelling clay, thermocol, salt, wax have been used for creating a hollow space inside the denture.

As a matter of fact, the role of gravity and the addition of weight to the mandibular complete denture in prosthesis retention has been stated by various authors.1,2 This is significant in patients with large defects in the maxillofacial region or severe residual ridge resorption. Hence, a hollow denture base might help in minimizing the weight of the prosthesis. ${ }^{3}$

Fattore et al. ${ }^{4}$ modified the double flask technique for obturator fabrication. ${ }^{5}$ He used a second drag so as to process a thin layer of resin surrounding the artificial teeth. The master cast was packed with heat polymerized resin simultaneously. Holt ${ }^{6}$ gave a technique of using a spacer, while the residual ridge was covered by a shim of resin. After indexing, the rest of the denture was fabricated by processing against the shim and spacer. It was finally removed, and the two portions were attached by means of autopolymerized acrylic resin. The indices helped in stabilization of the two halves.

However, the junction of two polymerized portions of the denture presented as a major limitation with these techniques. Microleakage into the hollow space in the denture can lead to bacterial accumulation. Furthermore, post insertion adjustment involving this junction can induce irregularities and the risk of microleakage increases. This method is technique sensitive, and determining the amount and thickness of resin covering the ridge and in the cope area presents as a tough task. ${ }^{3}$ This case report describes the fabrication of a hollow maxillary complete denture using silicone putty and a double flask technique.

In cases of increased inter arch space in completely edentulous patients, the double flask silicone putty technique is an accurate method to obtain the desired amount of hollow space that is required and thereby reducing the weight of the prosthesis.

It overcomes the disadvantage of weakening of the denture at certain sites due to an irregular hollow space created by compression of the material used during packing. However, a double flask technique makes it technique sensitive and hence needs to be done correctly to ensure maximum accuracy.

Financial or other competing interests: None.

Disclosure forms provided by the authors are available with the full text of this article at jemds.com.

\section{REFERENCES}

[1] Jacobson TE, Krol AJ. A contemporary review of the factors involved in complete denture retention, stability and support. Part I: retention. J Prosthet Dent 1983;49(1):5-15.

[2] Wormley JH, Brunton DA. Weighted mandibular dentures. J Prosthet Dent 1974;32(1):101-2.

[3] O'Sullivan M, Hansen N, Cronin RJ, et al. The hollow maxillary complete denture: a modified technique. J Prosthet Dent 2004;91(6):591-4.

[4] Fattore LD, Fine L, Edmonds DC. The hollow denture: an alternative treatment for atrophic maxillae. J Prosthet Dent 1988;59(4):514-6.

[5] Chalian VA, Barnett MO. A new technique for constructing a one-piece hollow obturator after partial maxillectomy. J Prosthet Dent 1972;28(4):448-53.

[6] Elliott DJ. The hollow bulb obturator: its fabrication using one denture flask. Quintessence Dent Technol 1983;7(1):13-4. 